\title{
Designing Hypolimnetic Aeration and Oxygenation Systems - A Review
}

\section{Supporting Information: Early Design Studies, Nomenclature, Tables, Figures, and Literature Cited}

\author{
VICKIE L. SINGLETON AND JOHN C. LITTLE* \\ Department of Civil and Environmental Engineering, Virginia Polytechnic Institute and State University, \\ Blacksburg, Virginia 24061, U.S.A. \\ * Corresponding author phone: (540) 231-8737; fax: (540) 231-7916; e-mail: jcl@vt.edu
}

\section{Early Design Studies}

Airlift Aerator. One of the first attempts to design airlift aerators was developed by Lorenzen and Fast (1). The primary objective was to size a compressor by determining the design air flow rate. The air flow rate is calculated using the hypolimnetic oxygen depletion rate and the induced water flow rate through the aerator. Based on general observations of full-lift aerators, the authors assumed that water reaching the top of the device is saturated with oxygen. The air flow rate required to induce this water flow rate is a function of the aerator dimensions. The available theoretical head results from the difference in density between the air-water mixture in the riser and the ambient lake water. It was also assumed that half of the theoretical head is used to convey water to the surface and that the remainder is dissipated in the downcomer. Lorenzen and Fast (1) provided practical guidance and information regarding the major variables that affect the performance of airlift aerators. However, the aerator sizing method presented makes a number of critical assumptions that are unverified.

The design method of Taggart and McQueen (2) involves determining the dimensions of the riser and downcomer when compressor capacity is known. The authors presented an empirically-based approach for establishing full-lift aerator specifications including diffuser depth, air flow rate, water flow rate, and riser and downcomer cross-sectional areas. Water flow rate is calculated using a correlation that was developed from a regression of data collected from 20 published experiments. To determine the optimum riser cross-sectional area, the authors assumed that the maximum induced water velocity is a function of the median estimated bubble rise velocity. The method of Taggart and McQueen (2) represents a simple, straightforward approach for the hydrodynamic design of airlift aerators. While the model did provide additional insight into aerator sizing, it lacks key elements. Oxygen transfer cannot be predicted by the model and the authors did not consider the effect of gas flux on induced water velocity. However, Taggart and McQueen (2) did account for gas flow when developing a correlation to calculate induced water flow rate as a function of volumetric air flow rate and riser depth.

Another empirical airlift aerator model was proposed by Ashley (3). In addition to aerator sizing, Ashley also discussed other practical design features including air supply, rated and actual air flow, and performance specifications. The model was derived from the work of Lorenzen and Fast (1) and Taggart and McQueen (2) as well as experience with a fullscale system. The model assumes that the induced water flow rate will completely satisfy the oxygen consumption in the hypolimnion measured during spring stratification. The model also requires an estimate of the increase in the dissolved oxygen (DO) concentration produced by the aerator. Determination of the DO increase is an important variable, and Ashley (3) suggested that this parameter may be difficult to predict. Ashley (3) presented a detailed, step-wise method for sizing an airlift aerator and provided useful information related to engineering aspects such as compressors, power supply, oxygen transfer efficiency, and oxygenation capacity. The model of Ashley (3) was field tested by Ashley and co-workers (4) (Supporting Information, Table 1), but the aeration system was unable to satisfy the hypolimnetic oxygen demand because the induced water velocity and oxygen input were overestimated during design. 
Little (5) developed a model to predict oxygen transfer in a full-lift hypolimnetic aerator by applying mass balance equations. Input parameters include aerator dimensions, volumetric air flow rate, diffuser depth, and ambient water conditions. To calculate water flow rate, an empirical correlation that is a function of superficial gas velocity (volumetric gas flow rate/entire riser cross-sectional area) and riser length was developed. The correlation was derived from the same data set used by Taggart and McQueen (2), except dependence on riser diameter was eliminated. Literature correlations, originally developed for bubble columns and airlift reactors, were used to estimate the mass transfer coefficient and gas holdup (volume fraction of gas in the bubblewater mixture) in the riser. The model assumes that gas holdup is small, water is in plug flow, and nitrogen transfer is negligible. The model proposed by Little (5) was the first attempt to develop a fundamental approach for predicting oxygen transfer in full-lift aerators, but the model relies on empirical correlations to calculate important variables. Also, nitrogen transfer can be significant for relatively deep systems and will subsequently affect bubble volume and bubble-size dependent properties including the rise velocity and mass transfer coefficient (6-8).

Bubble-Plume Diffuser. Rayyan and Speece (9) developed one of the earliest models to predict oxygen transfer and hydrodynamics of bubbleplumes in stratified environments. The model derivation extended the round or circular bubbleplume equations of Cederwall and Ditmars (10) to incorporate gas transfer and non-linear stratification. The model calculates maximum plume rise height, centerline velocity and nominal half-width, and density and temperature differences between the plume and the ambient water column. Conservation principles were applied to a circular plume to derive relationships for water, oxygen, momentum, buoyancy, and heat fluxes. Entrainment of ambient water is proportional to the plume centerline velocity (11), and the entrainment coefficient was set equal to 0.04 for low flow rates in the laboratory and 0.055 for higher flow rates in the field. The water and bubble spreading coefficients were 1.25 and 0.2 , respectively. Bubble size varies along the plume height and is a function of oxygen transfer and local hydrostatic pressure. The model of Rayyan and Speece (9) represents a significant advance in bubbleplume modeling because both oxygen transfer and stratification are considered. Although the model employs elements of the discrete-bubble approach, there are several key differences. The bubble rise velocity and mass transfer coefficients are not functions of bubble radius. Also, the transfer of gases other than oxygen is neglected. As mentioned previously, gaseous nitrogen exchange can significantly affect bubble size and related properties. The effect of salinity gradients on plume dynamics was not considered as was done in the Wüest et al. (7) model. Hydrodynamic predictions using the model were verified during both laboratory and field testing (Supporting Information, Table 1). However, the oxygen transfer portion of the model has not been validated with experimental data.

\section{Nomenclature}

$A_{d}$ : downcomer cross-sectional area, $\mathrm{m}^{2}$

$\mathrm{A}_{\mathrm{e}}$ : exit cross-sectional area, $\mathrm{m}^{2}$

$A_{r}$ : riser cross-sectional area, $\mathrm{m}^{2}$

b: circular plume radius for velocity and dissolved species, $\mathrm{m}$

$\mathrm{C}$ : aqueous-phase concentration, $\mathrm{mol} / \mathrm{m}^{3}$

$\mathrm{C}_{\mathrm{a}}$ : aqueous-phase concentration of ambient water, $\mathrm{mol} / \mathrm{m}^{3}$

$\mathrm{C}_{\mathrm{f}}$. Fanning friction factor, dimensionless

$\mathrm{D}_{\mathrm{d}}$ : downcomer diameter, $\mathrm{m}$

$\mathrm{D}_{\mathrm{r}}$ : riser diameter, $\mathrm{m}$

E: plume entrainment, $\mathrm{m}^{2} / \mathrm{s}$

$\mathrm{E}_{\mathrm{E}}$ : Energy loss due to local flow disturbances, $\mathrm{J} / \mathrm{s}$

$\mathrm{E}_{\mathrm{F}}$ : Energy loss due to wall friction, $\mathrm{J} / \mathrm{s}$

$\mathrm{E}_{\mathrm{I}}$ : Energy input due to isothermal gas expansion, $\mathrm{J} / \mathrm{s}$

$\mathrm{E}_{\mathrm{T}}$ : Energy loss due to friction at top of aerator, $\mathrm{J} / \mathrm{s}$

$\mathrm{E}_{\mathrm{W}}$ : Energy dissipation due to bubble wakes, $\mathrm{J} / \mathrm{s}$

$\mathrm{F}_{\mathrm{D}}$ : dissolved gas flux, mol/s

$\mathrm{F}_{\mathrm{G}}$ : gas flux, mol/s

$\mathrm{F}_{\mathrm{S}}$ : plume dissolved solids flux, $\mathrm{kg} / \mathrm{s}$

$\mathrm{F}_{\mathrm{T}}$ : plume temperature flux, ${ }^{\circ} \mathrm{C} \mathrm{m}^{3} / \mathrm{s}$

$\mathrm{g}$ : gravitational constant, $\mathrm{m} / \mathrm{s}^{2}$

$\mathrm{H}$ : Henry's constant, $\mathrm{mol} / \mathrm{m}^{3}$ bar

$\mathrm{hD}$ : aerated liquid height, $\mathrm{m}$

$\mathrm{h}_{\mathrm{L}}$ : unaerated liquid height, $\mathrm{m}$

$\mathrm{K}_{\mathrm{en}}$ : entrance loss coefficient, dimensionless

$\mathrm{K}_{\mathrm{ex}}$ : exit loss coefficient, dimensionless

$\mathrm{K}_{\mathrm{L}}$ : liquid-side mass transfer coefficient, $\mathrm{m} / \mathrm{s}$

$\mathrm{K}_{\mathrm{t}}$ : top section loss coefficient, dimensionless

$\mathrm{L}$ : linear plume length, $\mathrm{m}$

$\mathrm{L}_{\mathrm{d}}$ : length of downcomer, $\mathrm{m}$

M: plume momentum flux, $\mathrm{m}^{4} / \mathrm{s}^{2}$

$\mathrm{N}$ : number flux of bubbles, $1 / \mathrm{s}$

P: partial pressure, bar

$\mathrm{P}_{\text {atm: }}$ atmospheric pressure, $\mathrm{Pa}$

$P_{\text {tot: }}$ total pressure, bar 
Q: plume water volume flux, $\mathrm{m}^{3} / \mathrm{s}$

QG: volumetric gas flow rate at atmospheric

pressure, $\mathrm{m}^{3} / \mathrm{s}$

Qgas: actual volumetric gas flow rate at Speece

Cone inlet, $\mathrm{m}^{3} / \mathrm{s}$

$\mathrm{Q}_{\mathrm{W}}$ : volumetric water flow rate, $\mathrm{m}^{3} / \mathrm{s}$

$\mathrm{R}$ : ideal gas constant, $\mathrm{m}^{3} \mathrm{bar} / \mathrm{mol} \mathrm{K}$, or radius of

Speece Cone, $\mathrm{m}$

$\mathrm{R}_{1}$ : radius of top of Speece Cone, $\mathrm{m}$

$\mathrm{R}_{2}$ : radius of bottom of Speece Cone, $\mathrm{m}$

$\mathrm{r}$ : bubble radius, $\mathrm{m}$

S: salinity of plume water, $\mathrm{kg} / \mathrm{kg}$

$\mathrm{S}_{\mathrm{a}}$ : salinity of ambient water, $\mathrm{kg} / \mathrm{kg}$

$\mathrm{T}$ : temperature, ${ }^{\circ} \mathrm{C}$ or $\mathrm{K}$

$\mathrm{T}_{\mathrm{a}}$ : temperature of ambient water, ${ }^{\circ} \mathrm{C}$

$\mathrm{T}_{\mathrm{p}}$ : temperature of plume water, ${ }^{\circ} \mathrm{C}$

$\mathrm{U}_{\mathrm{G}}$ : average superficial gas velocity, $\mathrm{m} / \mathrm{s}$

$\mathrm{ULd}_{\mathrm{Ld}}$ : superficial water velocity in downcomer, $\mathrm{m} / \mathrm{s}$

ULe: superficial water velocity in exit, $\mathrm{m} / \mathrm{s}$

$\mathrm{U}_{\mathrm{Lr}}$ : superficial water velocity in riser, $\mathrm{m} / \mathrm{s}$

$\mathrm{V}_{\text {Lr}}$ : actual water velocity in riser, $\mathrm{m} / \mathrm{s}$

v: actual water velocity, $\mathrm{m} / \mathrm{s}$

$\mathrm{vb}$ : bubble rise velocity, $\mathrm{m} / \mathrm{s}$
$\mathrm{W}$ : linear plume width, $\mathrm{m}$

$\mathrm{y}$ : gaseous-phase concentration, $\mathrm{mol} / \mathrm{m}^{3}$

$\mathrm{z}$ : depth, $\mathrm{m}$ (defined as positive downwards for Speece Cone)

$\triangle \mathrm{DO}$ : change in dissolved oxygen concentration, $\mathrm{g} / \mathrm{m}^{3}$

\section{Greek variables}

$\alpha$ : entrainment coefficient, dimensionless

$\varepsilon$ : gas holdup, dimensionless

$\lambda$ : plume radius or spreading ratio, dimensionless

$\rho:$ average density of air-water mixture in airlift aerator, $\mathrm{kg} / \mathrm{m}^{3}$

$\rho_{\mathrm{L}}$ : water density, $\mathrm{kg} / \mathrm{m}^{3}$

$\rho_{\mathrm{a}}$ : ambient water density, $\mathrm{kg} / \mathrm{m}^{3}$

$\rho_{\mathrm{p}}$ : plume bubble-water mixture density, $\mathrm{kg} / \mathrm{m}^{3}$

$\rho_{\mathrm{w}}$ : density of plume water, $\mathrm{kg} / \mathrm{m}^{3}$

\section{Subscripts}

a: ambient

i: individual chemical species (oxygen or nitrogen)

r: riser

O: oxygen

$\mathrm{N}$ : nitrogen 


\section{Tables}

Table 1. Summary of selected hypolimnetic aeration and oxygenation installations documented in the literature. Both experimental and permanent units are included.

\begin{tabular}{|c|c|c|c|c|c|c|}
\hline Waterbody & $\begin{array}{l}\text { Maximum } \\
\text { Depth } \\
\text { (m) }\end{array}$ & $\begin{array}{l}\text { Volume } \\
\left(10^{6} \mathrm{~m}^{3}\right)\end{array}$ & Oxygenator Type & Year Installed & $\begin{array}{l}\text { Oxygen } \\
\text { Addition } \\
(\mathrm{kg} / \mathrm{d})\end{array}$ & References \\
\hline Wahnbach Reservoir, Germany & 45 & 42 & full-lift aerator & 1966 & 1,560 & $(12,13)$ \\
\hline Mirror Lake, Wisconsin & 13 & 0.40 & full-lift aerator & 1972 & 111 & (14) \\
\hline Silver Lake, Wisconsin & 12 & & full-lift aerator & 1972 & 85 & (14) \\
\hline Larson Lake, Wisconsin & 12 & 0.19 & full-lift aerator & 1973 & 26 & (14) \\
\hline Lake Waccabuc, New York & 13 & 4.1 & partial-lift aerator & 1973 & 350 & $(15)$ \\
\hline Ottoville Quarry, Ohio & 18 & 0.063 & side stream pumping & 1973 & 14 & (16) \\
\hline Spruce Knob Lake, West Virginia & 6 & & full-lift aerator & 1974 & 49 & $(17)$ \\
\hline Clark Hill Reservoir, Georgia & & 3,096 & bubble-plume diffuser & 1975 & 54,400 & (9) \\
\hline Lake Ghirla, Italy & 14 & 2.0 & $\begin{array}{l}\text { submerged pumping } \\
\text { oxygenation system }^{\text {a }}\end{array}$ & 1976 & 0.13 & $(18,19)$ \\
\hline Lake Nantua, France & 42 & & side stream oxygen injection & 1976 & $200-250$ & $(20)$ \\
\hline Black Lake, British Columbia & 9 & 0.18 & full-lift aerator & 1978 & & $(21)$ \\
\hline Tory Lake, Ontario & 10 & 0.055 & full-lift aerator & 1978 & & $(22,23)$ \\
\hline Lake Särkinen, Finland & 17 & 2.5 & Mixox aerator & 1980 & & (24) \\
\hline Lake St. George, Ontario & 16 & & full-lift aerator & 1980 & & $(25,26)$ \\
\hline Lake Tegal, Germany & 16 & 24.6 & Limnox partial-lift aerator & 1980 & 4,500 & $(27)$ \\
\hline We $\beta$ linger See, Germany & 12 & 1.0 & Limno full-lift aerator & 1981 & 120 & $(28)$ \\
\hline Lake Baldegg, Switzerland & 66 & 176 & bubble-plume diffuser & 1982 & $3,000-4,500$ & $(7,29)$ \\
\hline Lake Pyhäjärvi, Finland & 42 & & Mixox aerator & 1983 & 1,300 & $(24)$ \\
\hline Lake Sempach, Switzerland & 87 & 662 & bubble-plume diffuser & 1984 & 3,000 & (29) \\
\hline Lake Hald, Denmark & 31 & 44 & bubble-plume diffuser & & 575 & $(30)$ \\
\hline $\begin{array}{l}\text { Richard B. Russell Reservoir, } \\
\text { Georgia }\end{array}$ & 47 & 1,270 & bubble-plume diffuser & 1985 & 200,000 & $(19,31)$ \\
\hline Glen Lake, British Columbia & 13 & & full-lift aerator & 1986 & 40 & (4) \\
\hline Lake Hallwil, Switzerland & 47 & 285 & bubble-plume diffuser & 1986 & $1,000-7,100$ & $(32)$ \\
\hline Lake Kallvesi, Finland & 38 & & Mixox aerator & 1986 & & (33) \\
\hline Medical Lake, Washington & 18 & 6.2 & LIMNO partial-lift aerator & 1986 & 225 & (34) \\
\hline St. Mary Lake, British Columbia & 9.1 (mean) & & full-lift aerator & 1986 & $\begin{array}{l}311 \\
512 \text { (after } \\
\text { retrofit) }\end{array}$ & $(35,36)$ \\
\hline Lake Shenipsit, Connecticut & 21 & 12.3 & layer aeration & 1987 & & $(37,38)$ \\
\hline Lake Muggesfelde, Germany & 21 & 21 & TIBEAN full-lift aerator & 1987 & 500 & (39) \\
\hline
\end{tabular}




\begin{tabular}{|c|c|c|c|c|c|c|}
\hline Waterbody & $\begin{array}{l}\text { Maximum } \\
\text { Depth } \\
(\mathrm{m})\end{array}$ & $\begin{array}{l}\text { Volume } \\
\left(10^{6} \mathrm{~m}^{3}\right)\end{array}$ & Oxygenator Type & Year Installed & $\begin{array}{l}\text { Oxygen } \\
\text { Addition } \\
(\mathrm{kg} / \mathrm{d}) \\
\end{array}$ & References \\
\hline Amisk Lake, Alberta & $\begin{array}{c}34 \text { (north } \\
\text { basin) }\end{array}$ & $\begin{array}{c}25 \text { (north } \\
\text { basin) }\end{array}$ & bubble-plume diffuser & 1988 & $750-1,000$ & $(40,41)$ \\
\hline Lake Huruslahti, Finland & 26 & & Mixox aerator & 1990 & & (33) \\
\hline Lake Krupunder, Germany & 10.5 & 0.28 & TIBEAN full-lift aerator & 1990 & 80 & $(42)$ \\
\hline Medical Lake, Washington & 18 & 6.2 & full-lift aerator & 1990 & 500 & $(34)$ \\
\hline Lake Prince, Virginia & 10 & 13.9 & full-lift aerator & 1991 & 4,100 & $(43,44)$ \\
\hline Newman Lake, Washington & 10 & 28.6 & Speece Cone & 1992 & 2,000 & $(45,46)$ \\
\hline Camanche Reservoir, California & 41 & 545 & Speece Cone & 1993 & 9,000 & $(47)$ \\
\hline Douglas Dam, Tennessee & 38 & 1700 & bubble-plume diffuser & 1993 & 100,000 & $(48)$ \\
\hline Lake Western Branch, Virginia & 11 & 24.4 & full-lift aerator & 1993 & 6,600 & $(43,44)$ \\
\hline Lake Stevens, Washington & 44 & & full-lift aerator & 1994 & 2,900 & $(49)$ \\
\hline Tombigbee River, Alabama & 11 & $\mathrm{n} / \mathrm{a}$ & U-tube & & 23,600 & $(50)$ \\
\hline Heart Lake, Ontario & 10.9 & 0.78 & full-lift oxygenator & 1995 & $140-200$ & $(51)$ \\
\hline Whittaker Lake, Ontario & 11 & 0.39 & full-lift oxygenator & 1995 & $140-200$ & $(51)$ \\
\hline Spring Hollow Reservoir, Virginia & 55 & 7.2 & bubble-plume diffuser & 1998 & 250 & $(52,53)$ \\
\hline $\begin{array}{l}\text { Upper San Leandro Reservoir, } \\
\text { California }\end{array}$ & & 51 & bubble-plume diffuser & 2002 & 9,000 & $(53,54)$ \\
\hline
\end{tabular}

as classified by Beutel and Horne (1999) 
Table 2. Correlation equations for Henry's constant, mass transfer coefficient, and bubble rise velocity (7).

\begin{tabular}{ll}
\hline \multicolumn{1}{c}{ Equation } & \multicolumn{1}{c}{ Range } \\
\hline $\mathrm{H}_{\mathrm{O}}=2.125-5.021 \times 10^{-2} \mathrm{~T}+5.77 \times 10^{-4} \mathrm{~T}^{2}$ & (T in Celsius) \\
$\mathrm{H}_{\mathrm{N}}=1.042-2.450 \times 10^{-2} \mathrm{~T}+3.171 \times 10^{-4} \mathrm{~T}^{2}$ & \\
& \\
$\mathrm{~K}_{\mathrm{L}}=0.6 \mathrm{r}$ & $\mathrm{r}<6.67 \times 10^{-4} \mathrm{~m}$ \\
$\mathrm{~K}_{\mathrm{L}}=4 \times 10^{-4}$ & $\mathrm{r} \geq 6.67 \times 10^{-4} \mathrm{~m}$ \\
& \\
$\mathrm{v}_{\mathrm{b}}=4474 \mathrm{r}^{1.357}$ & $\mathrm{r}<7 \times 10^{-4} \mathrm{~m}$ \\
$\mathrm{v}_{\mathrm{b}}=0.23$ & $7 \times 10^{-4} \leq \mathrm{r}<5.1 \times 10^{-3} \mathrm{~m}$ \\
$\mathrm{v}_{\mathrm{b}}=4.202 \mathrm{r}^{0.547}$ & $\mathrm{r} \geq 5.1 \times 10^{-3} \mathrm{~m}$ \\
\hline
\end{tabular}


Table 3. Non-linear differential flux equations of the airlift aerator (44) and Speece Cone (55) models.

\begin{tabular}{ll}
$\begin{array}{l}\text { Dissolved gas flux } \\
\text { (oxygen and nitrogen) }\end{array}$ & $\frac{\mathrm{dF}_{\mathrm{D}_{\mathrm{i}}}}{\mathrm{dz}}=\mathrm{K}_{\mathrm{L}}\left(\mathrm{H}_{\mathrm{i}} \mathrm{P}_{\mathrm{i}}-\mathrm{C}_{\mathrm{i}}\right) \frac{4 \pi \mathrm{r}^{2} \mathrm{~N}}{\left(\mathrm{v}+\mathrm{v}_{\mathrm{b}}\right)(1-\varepsilon)}$ \\
$\begin{array}{l}\text { Gas flux } \\
\text { (oxygen and nitrogen) }\end{array}$ & $\frac{\mathrm{dF}_{\mathrm{G}_{\mathrm{i}}}}{\mathrm{dz}}=-\mathrm{K}_{\mathrm{L}}\left(\mathrm{H}_{\mathrm{i}} \mathrm{P}_{\mathrm{i}}-\mathrm{C}_{\mathrm{i}}\right) \frac{4 \pi \mathrm{r}^{2} \mathrm{~N}}{\mathrm{v}+\mathrm{v}_{\mathrm{b}}}$ \\
\hline
\end{tabular}


Table 4. Key variables and water flow rate equations of the airlift aerator model $(44,56)$.

\begin{tabular}{|c|c|}
\hline $\begin{array}{l}\text { Dissolved gas flux } \\
\text { (oxygen and nitrogen) } \\
\text { Gas flux } \\
\text { (oxygen and nitrogen) }\end{array}$ & $\begin{array}{l}\mathrm{F}_{\mathrm{D}_{\mathrm{i}}}=\mathrm{A}_{\mathrm{r}}(1-\varepsilon) \mathrm{vC}_{\mathrm{i}} \\
\mathrm{F}_{\mathrm{G}_{\mathrm{i}}}=\mathrm{A}_{\mathrm{r}}\left(\mathrm{v}+\mathrm{v}_{\mathrm{b}}\right) \mathrm{y}_{\mathrm{i}}\end{array}$ \\
\hline Bubble radius & $\mathrm{r}=\left[\frac{3 \varepsilon \mathrm{A}_{\mathrm{r}}\left(\mathrm{v}+\mathrm{v}_{\mathrm{b}}\right)}{4 \pi \mathrm{N}}\right]^{\frac{1}{3}}$ \\
\hline $\begin{array}{l}\text { Energy balance for water } \\
\text { flow rate }\end{array}$ & $\mathrm{E}_{\mathrm{I}}=\mathrm{E}_{\mathrm{W}}+\mathrm{E}_{\mathrm{F}}+\mathrm{E}_{\mathrm{E}}+\mathrm{E}_{\mathrm{T}}$ \\
\hline $\begin{array}{l}\text { Energy input due to } \\
\text { isothermal gas expansion }\end{array}$ & $\mathrm{E}_{\mathrm{I}}=\mathrm{Q}_{\mathrm{G}} \mathrm{P}_{\mathrm{atm}} \ln \left(1+\frac{\rho \mathrm{gh}_{\mathrm{D}}}{\mathrm{P}_{\mathrm{atm}}}\right)$ \\
\hline $\begin{array}{l}\text { Energy dissipation due to } \\
\text { bubble wakes }\end{array}$ & $\mathrm{E}_{\mathrm{W}}=\rho_{\mathrm{L}} \mathrm{gh}_{\mathrm{L}} \mathrm{A}_{\mathrm{r}} \varepsilon_{\mathrm{r}} \mathrm{v}_{\mathrm{b}}$ \\
\hline $\begin{array}{l}\text { Energy loss due to wall } \\
\text { friction }\end{array}$ & $E_{F}=2 C_{f} \rho_{L} U_{L r}\left(U_{L r}+U_{G}\right) \frac{h_{D}}{D_{r}}\left(U_{L r} A_{r}\right)+2 C_{f} \rho_{L} \frac{L_{d}}{D_{d}}\left(U_{L d}^{3} A_{d}\right)$ \\
\hline $\begin{array}{l}\text { Energy loss due to local flow } \\
\text { disturbances }\end{array}$ & $\mathrm{E}_{\mathrm{E}}=\frac{1}{2} \rho_{\mathrm{L}}\left[\mathrm{V}_{\mathrm{Lr}}^{3} \mathrm{~K}_{\mathrm{en}} \mathrm{A}_{\mathrm{r}}\left(1-\varepsilon_{\mathrm{r}}\right)+\mathrm{U}_{\mathrm{Le}}^{3} \mathrm{~K}_{\mathrm{ex}} \mathrm{A}_{\mathrm{e}}\right]$ \\
\hline $\begin{array}{l}\text { Energy loss due to friction at } \\
\text { top of aerator }\end{array}$ & $\mathrm{E}_{\mathrm{T}}=\frac{1}{2} \rho_{\mathrm{L}} \mathrm{V}_{\mathrm{Lr}}^{3} \mathrm{~K}_{\mathrm{t}} \mathrm{A}_{\mathrm{r}}\left(1-\varepsilon_{\mathrm{r}}\right)$ \\
\hline
\end{tabular}


Table 5. Key variables of the Speece Cone model (55).

\begin{tabular}{ll}
\hline Molar dissolved gas flow rate & $\mathrm{F}_{\mathrm{D}_{\mathrm{i}}}=\pi \mathrm{R}^{2} \mathrm{v} \mathrm{C}_{\mathrm{i}}$ \\
Molar gas flow rate & $\mathrm{F}_{\mathrm{G}_{\mathrm{i}}}=\pi \mathrm{R}^{2}\left(\mathrm{v}+\mathrm{v}_{\mathrm{b}}\right) \mathrm{y}_{\mathrm{i}}$ \\
Cone radius & $\mathrm{R}=\frac{\mathrm{R}_{2}-\mathrm{R}_{1}}{\mathrm{~h}} \mathrm{z}+\mathrm{R}_{1}$ \\
Actual water velocity & $\mathrm{v}=\frac{\mathrm{Q}_{\mathrm{w}}}{\pi}\left(\frac{\mathrm{R}_{2}-\mathrm{R}_{1}}{\mathrm{~h}} \mathrm{z}+\mathrm{R}_{1}\right)^{-2}\left(\frac{1}{1-\varepsilon}\right)$ \\
\hline
\end{tabular}


Table 6. Speece Cone performance at varying depths predicted using assumed cone dimensions and operational parameters (55).

\begin{tabular}{ccccc}
\hline $\begin{array}{c}\text { Depth } \\
(\mathrm{m})\end{array}$ & $\begin{array}{c}\mathrm{Q}_{\mathrm{gas}} \\
(\mathrm{L} / \mathrm{s})\end{array}$ & $\begin{array}{c}\Delta \mathrm{DO} \\
\left(\mathrm{g} / \mathrm{m}^{3}\right)\end{array}$ & $\begin{array}{c}\text { Total Oxygen } \\
\text { Transfer } \\
(\mathrm{kg} / \text { day })\end{array}$ & $\begin{array}{c}\text { Bubble Residence } \\
\text { Time } \\
(\mathrm{s})\end{array}$ \\
\hline 0 & 20 & 17 & 2,200 & 107 \\
10 & 40 & 33 & 4,300 & 75 \\
20 & 60 & 50 & 6,400 & 69 \\
30 & 80 & 66 & 8,600 & 66 \\
40 & 100 & 83 & 10,700 & 64 \\
50 & 120 & 101 & 12,800 & 62 \\
\hline
\end{tabular}


Table 7. Key variables of the circular (7) and linear (57) bubble-plume models.

\begin{tabular}{lll}
\hline \multicolumn{1}{c}{ Variable } & \multicolumn{1}{c}{ Circular Bubble Plume } & \multicolumn{1}{c}{ Linear Bubble Plume } \\
\hline Entrainment & $\mathrm{E}=2 \alpha \pi \mathrm{bv}$ & $\mathrm{E}=2(\mathrm{~L}+\mathrm{W}) \alpha \mathrm{v}$ \\
Plume water volume flux & $\mathrm{Q}=\pi \mathrm{b}^{2} \mathrm{v}$ & $\mathrm{Q}=\mathrm{LWv}$ \\
Momentum flux & $\mathrm{M}=\pi \mathrm{b}^{2} \mathrm{v}^{2}$ & $\mathrm{M}=\mathrm{LWv}^{2}$ \\
Temperature flux & $\mathrm{F}_{\mathrm{T}}=\mathrm{QT}_{\mathrm{p}}$ & $\mathrm{F}_{\mathrm{T}}=\mathrm{QT}_{\mathrm{p}}$ \\
Dissolved solids flux & $\mathrm{F}_{\mathrm{S}}=\mathrm{QS} \rho_{\mathrm{w}}$ & $\mathrm{F}_{\mathrm{s}}=\mathrm{QS} \rho_{\mathrm{w}}$ \\
Dissolved $\mathrm{O}_{2}$ and $\mathrm{N}_{2}$ fluxes & $\mathrm{F}_{\mathrm{D}_{\mathrm{i}}}=\mathrm{QC}_{\mathrm{i}}$ & $\mathrm{F}_{\mathrm{D}_{\mathrm{i}}}=\mathrm{QC} \mathrm{i}$ \\
Gaseous $\mathrm{O}_{2}$ and $\mathrm{N}_{2}$ fluxes & $\mathrm{F}_{\mathrm{G}_{\mathrm{i}}}=\pi \mathrm{b}^{2} \lambda^{2}\left(\mathrm{v}+\mathrm{v}_{\mathrm{b}}\right) \mathrm{y}_{\mathrm{i}}$ & $\mathrm{F}_{\mathrm{G}_{\mathrm{i}}}=\lambda \mathrm{W}[\mathrm{L}-(\mathrm{W}-\lambda \mathrm{W})]\left(\mathrm{v}+\mathrm{v}_{\mathrm{b}}\right) \mathrm{y}_{\mathrm{i}}$ \\
\hline
\end{tabular}


Table 8. Non-linear differential flux equations of the circular (7) and linear (57) bubble-plume models.

\begin{tabular}{ll} 
Water volume flux & $\frac{\mathrm{dQ}}{\mathrm{dz}}=\mathrm{E}$ \\
$\begin{array}{l}\text { Momentum flux } \\
\text { (circular bubble plume) }\end{array}$ & $\frac{\mathrm{dM}}{\mathrm{dz}}=\frac{\rho_{\mathrm{a}}-\rho_{\mathrm{p}}}{\rho_{\mathrm{p}}} \mathrm{g} \pi \mathrm{b}^{2} \lambda^{2}+\frac{\rho_{\mathrm{a}}-\rho_{\mathrm{w}}}{\rho_{\mathrm{p}}} \mathrm{g} \pi \mathrm{b}^{2}\left(1-\lambda^{2}\right)$ \\
$\begin{array}{l}\text { Momentum flux } \\
\text { (linear bubble plume) }\end{array}$ & $\frac{\mathrm{dM}}{\mathrm{dz}}=\frac{\rho_{\mathrm{a}}-\rho_{\mathrm{w}}}{\rho_{\mathrm{p}}} \mathrm{gLW}+\frac{\rho_{\mathrm{w}}-\rho_{\mathrm{p}}}{\rho_{\mathrm{p}}} \mathrm{g} \lambda \mathrm{W}[\mathrm{L}-\mathrm{W}(1-\lambda)]$ \\
$\begin{array}{l}\text { Temperature flux } \\
\text { Salinity flux }\end{array}$ & $\frac{\mathrm{dF} \mathrm{T}_{\mathrm{S}}}{\mathrm{dz}}=\mathrm{ET}_{\mathrm{a}}$ \\
$\begin{array}{l}\text { Dissolved gas flux } \\
\text { (oxygen and nitrogen) }\end{array}$ & $\frac{\mathrm{dz} \rho_{\mathrm{a}} \mathrm{S}_{\mathrm{a}}}{\mathrm{dz}}=\mathrm{EC}_{\mathrm{ai}}+\mathrm{K}_{\mathrm{L}}\left(\mathrm{H}_{\mathrm{i}} \mathrm{P}_{\mathrm{i}}-\mathrm{C}_{\mathrm{i}}\right) \frac{4 \pi \mathrm{r}^{2} \mathrm{~N}}{\mathrm{v}+\mathrm{v}_{\mathrm{b}}}$ \\
$\begin{array}{l}\text { Gas flux } \\
\text { (oxygen and nitrogen) }\end{array}$ & $\frac{\mathrm{dF}_{\mathrm{Gi}}}{\mathrm{dz}}=-\mathrm{K}_{\mathrm{L}}\left(\mathrm{H}_{\mathrm{i}} \mathrm{P}_{\mathrm{i}}-\mathrm{C}_{\mathrm{i}}\right) \frac{4 \pi \mathrm{r}^{2} \mathrm{~N}}{\mathrm{v}+\mathrm{v}_{\mathrm{b}}}$ \\
\hline
\end{tabular}




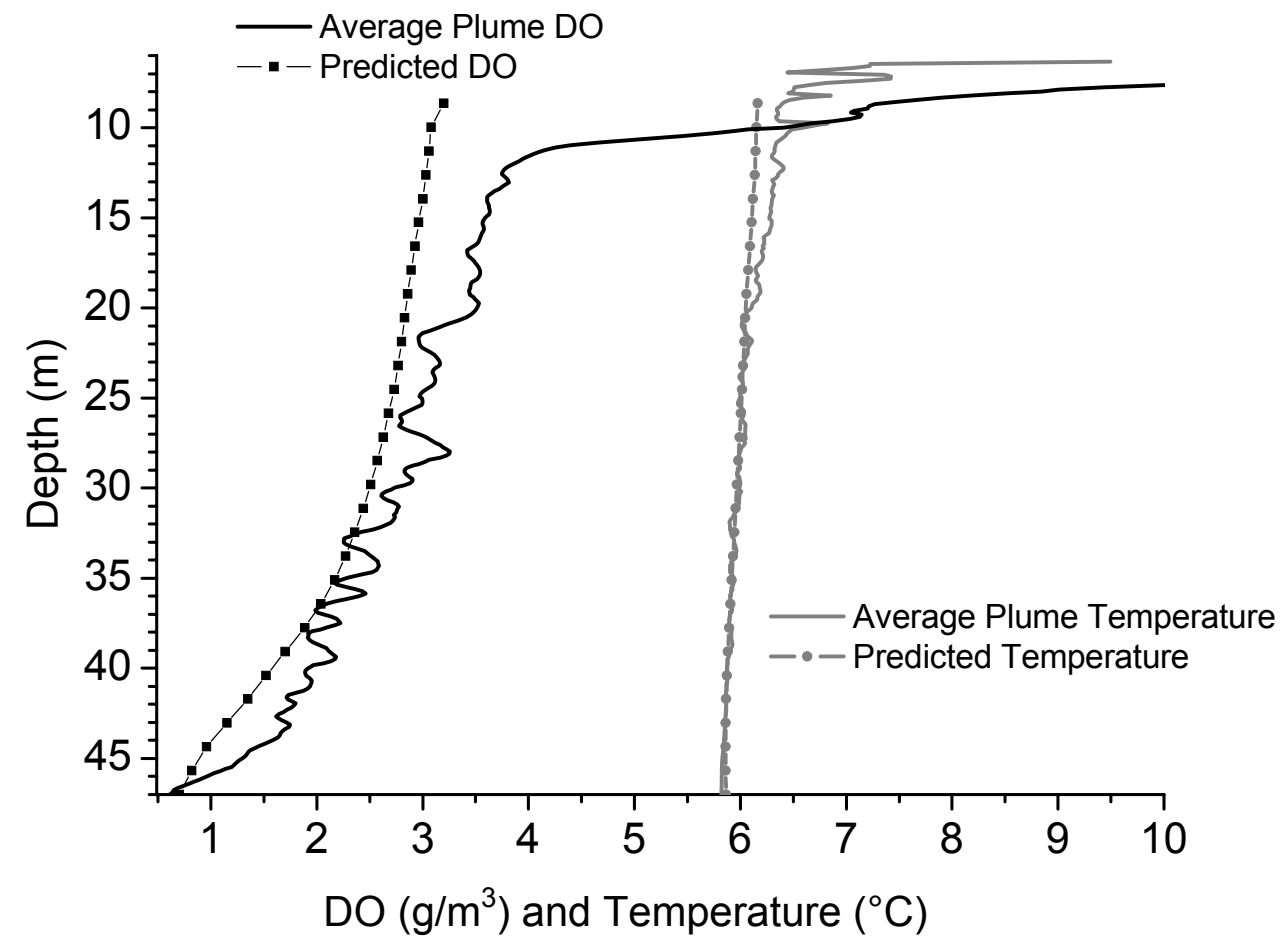

Figure 1. Averaged measured in-plume temperature and dissolved oxygen (DO) profile and circular bubble-plume model predictions for Lake Hallwil, Switzerland (32). 

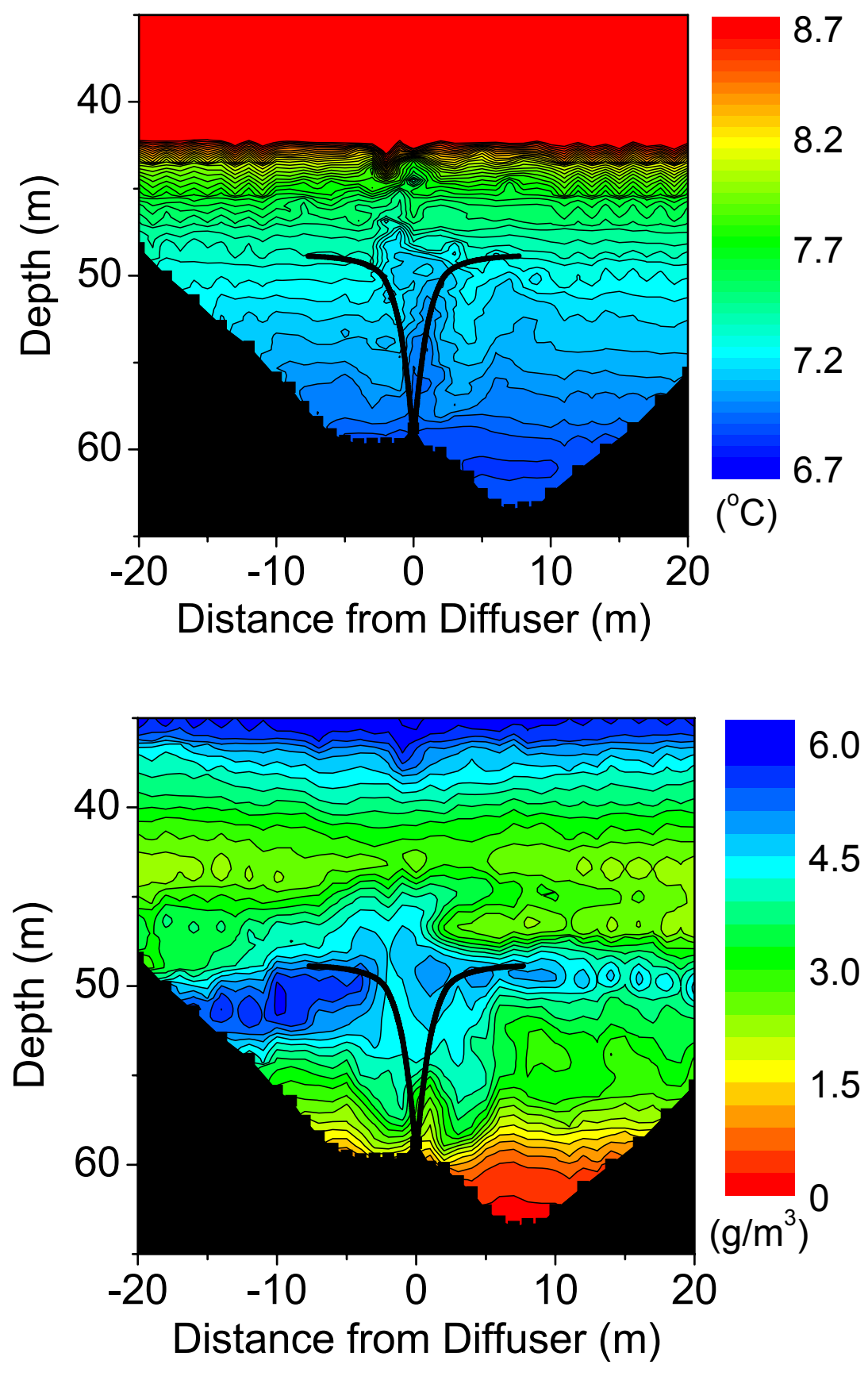

Figure 2. Measured temperature (top) and dissolved oxygen (bottom) contours with linear bubble-plume model predictions for diffuser operation with pure oxygen on 23 October 2004 in Spring Hollow Reservoir, VA, U.S.A. (57). 

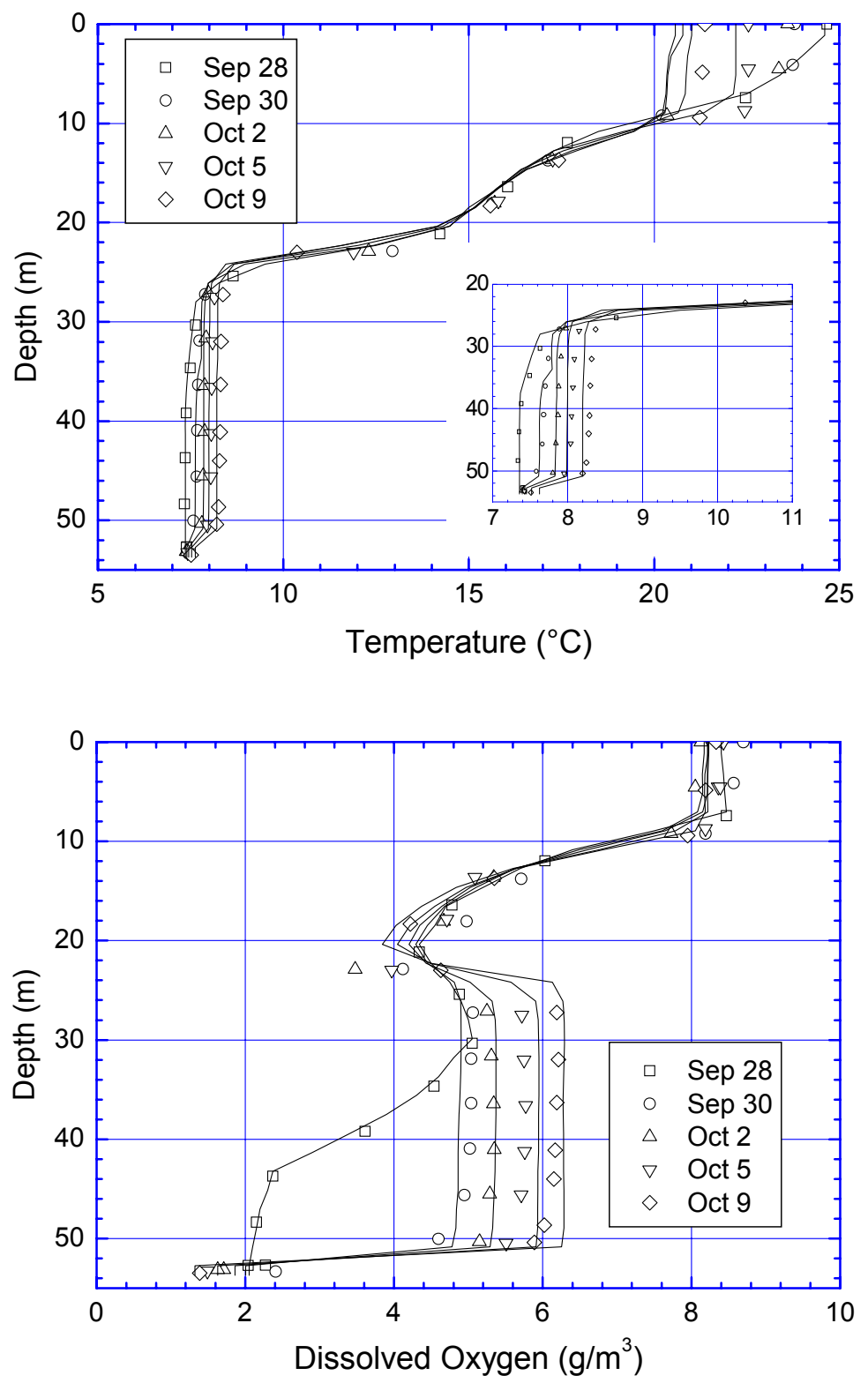

Figure 3. Measured temperature (top) and dissolved oxygen (bottom) profiles with coupled bubble plume-reservoir model predictions for linear diffuser operation in Spring Hollow Reservoir, VA, U.S.A. during 1997 (58). 


\section{Literature Cited}

(1) Lorenzen, M. W.; Fast, A. W. A Guide to Aeration/Circulation Techniques for Lake Management; EPA-600/3-77-004, U.S. Environmental Protection Agency Ecol. Res. Serv.: 1977.

(2) Taggart, C. T.; McQueen, D. J. A model for the design of hypolimnetic aerators. Water Res. 1982, 16, 949-956.

(3) Ashley, K. I. Hypolimnetic aeration: Practical design and application. Water Res. 1985, 19, 735-740.

(4) Ashley, K. I.; Hay, S.; Scholten, G. H. Hypolimnetic aeration: Field test of the empirical sizing method. Water Res. 1987, 21, 223-227.

(5) Little, J. C. Hypolimnetic aerators: Predicting oxygen transfer and hydrodynamics. Water Res. 1995, 29, 2475-2482.

(6) Clift, R.; Grace, J. R.; Weber, M. E. Bubble, Drops, and Particles New York, NY, 1978.

(7) Wüest, A.; Brooks, N. H.; Imboden, D. M. Bubble plume modeling for lake restoration. Water Resour. Res. 1992, 28, 3235-3250.

(8) Leifer, I.; Patro, R. K. The bubble mechanism for methane transport from the shallow sea bed to the surface: A review and sensitivity study. Cont. Shelf Res. 2002, 22, 2409-2428.

(9) Rayyan, F.; Speece, R. E. Hydrodynamics of bubble plumes and oxygen absorption in stratified impoundments. Prog. Water Technol. 1977, 9, 129-142.

(10) Cederwall, K.; Ditmars, J. D. Analysis of AirBubble Plumes; KH-R-24, W. M. Keck Laboratory of Hydraulics and Water Resources, Division of Engineering and Applied Science, California Institute of Technology: 1970.

(11) Morton, B. R.; Taylor, G. I.; Turner, J. S. Turbulent gravitational convection from maintained and instantaneous sources. Proc. of the Royal Soc. of London. Ser. A, Mathematical and Phys. Sci. 1956, 234, 1-23.

(12) Bernhardt, H. Aeration of Wahnbach Reservoir without changing the temperature profile. $J$. Am. Water Works Assoc. 1967, 59, 943-964.

(13) Bernhardt, H.; Wilhelms, A. Hypolimnetic aeration as a means of controlling redox processes on the bottom of a eutrophic reservoir. 1975, 19, 1957-1959.

(14) Wirth, T. L.; Knauer, D. R.; Smith, S. A. Total and hypolimnetic aeration of lakes in Wisconsin. Verh. Internat. Verein. Limnol. 1975, 19, 1960-1970.
(15) Fast, A. W.; Dorr, V. A.; Rosen, R. J. A submerged hypolimnion aerator. Water Resour. Res. 1975, 11, 287-293.

(16) Fast, A. W.; Overholtz, W. J.; Tubb, R. A. Hypolimnetic oxygenation using liquid oxygen. Water Resour. Res. 1975, 11, 294-299.

(17) Hess, L. The effect of the 1st year of artificial hypolimnion aeration on oxygen, temperature, and the depth distribution of rainbow trout Salmo gairdneri in Spruce Knob Lake, West Virginia, U.S.A. In Proceedings of the West Virginia Academy of Science: 1975, 176-183.

(18) Bianucci, G.; Bianucci, E. R. Oxygenation of a polluted lake in northern Italy. Effluent and Water Treatment J. 1979, 19, 117-127.

(19) Beutel, M. W.; Horne, A. J. A review of the effects of hypolimnetic oxygenation on lake and reservoir water quality. Lake Reservoir Manage. 1999, 15, 285-297.

(20) Barroin, G. The sewage diversion and hypolimnetic oxygenation projects: Techniques and effectiveness. Arch. Hydrobiol. Beih. 1994, 41, 17-31.

(21) Ashley, K. I.; Hall, K. J. Factors influencing oxygen transfer in hypolimnetic aeration systems. Internat. Vereinigung fuer Theoret. und Angewandte Limnol. 1990, 24, 179-183.

(22) Taggart, C. T.; McQueen, D. J. Hypolimnetic aeration of a small eutrophic kettle lake: Physical and chemical changes. Arch. Hydrobiol. 1981, 91, 150-180.

(23) Taggart, C. T. Hypolimnetic aeration and zooplankton distribution: A possible limitation to the restoration of cold-water fish production. Can. J. Fish. Aquat. Sci. 1984, 41, 191-198.

(24) Lappalainen, K. M. Positive changes in oxygen and nutrient contents in two Finnish lakes induced by Mixox hypolimnetic oxygenation method. Internat. Vereinigung fuer Theoret. und Angewandte Limnol. 1994, 25, 2510-2513.

(25) McQueen, D. J.; Lean, D. R. S. Hypolimnetic aeration and dissolved gas concentrations: Enclosure experiments. Water Res. 1983, 17, 1781-1790.

(26) McQueen, D. J.; Lean, D. R. S.; Charlton, M. N. Effects of hypolimnetic aeration on ironphosphorus interactions. Water Res. 1986, 20, 1129-1135.

(27) Lindenschmidt, K. E.; Hamblin, P. F. Hypolimnetic aeration in Lake Tegel, Berlin. Water Res. 1997, 31, 1619-1628.

(28) Steinberg, C.; Arzet, K. Impact of hypolimnetic aeration on abiotic and biotic conditions in a small kettle lake. Environ. Technol. Letters 1984, 5, 151-162. 
(29) Gachter, R.; Wehrli, B. Ten years of artificial mixing and oxygenation: No effect on the internal phosphorus loading of two eutrophic lakes. Environ. Sci. Technol. 1998, 32, 36593665.

(30) Søndergaard, M.; Jeppesen, E.; Jensen, P. J.; Lauridsen, T. Lake restoration in Denmark. Lakes Reserv.: Res. Manage. 2000, 5, 151-159.

(31) Mauldin, G.; Miller, R.; Gallagher, J.; Speece, R. E. Injecting an oxygen fix. Civ. Eng. CEWRA9 1988, 58, 54-56.

(32) McGinnis, D. F.; Lorke, A.; Wüest, A.; Stöckli, A.; Little, J. C. Interaction between a bubble plume and the near-field in a stratified lake. Water Resour. Res. 2004, 40.

(33) Matinvesi, J. The change of sediment composition during recovery of two Finnish lakes induced by waste water purification and lake oxygenation. Hydrobiol. 1996, 335, 193202.

(34) Soltero, R. A.; Sexton, L. M.; Ashley, K. I.; McKee, K. O. Partial and full lift hypolimnetic aeration of Medical Lake, WA to improve water quality. Water Res. 1994, 28, 2297-2308.

(35) Ashley, K. I. Hypolimnetic aeration research in British Columbia. Verh. Internat. Verein. Limnol. 1988, 23, 215-219.

(36) Ashley, K. I. Recent advances in hypolimnetic aeration design. Verh. Internat. Verein. Limnol. 2000, 27, 2256-2260.

(37) Kortmann, R. W. Oligotrophication of Lake Shenipsit by layer aeration. Lake Reserv. Manage. 1994, 9, 94-97.

(38) Kortmann, R. W.; Knoecklein, G. W.; Bonnell, C. H. Aeration of stratified lakes: Theory and practice. Lake Reserv. Manage. 1994, 8, 99120.

(39) Jaeger, D. TIBEAN: A new hypolimnetic water aeration plant. Internat. Vereinigung fuer Theoret. und Angewandte Limnol. 1990, 24, 184-187.

(40) Prepas, E. E.; Burke, J. M. Effects of hypolimnetic oxygenation on water quality in Amisk Lake, Alberta, a deep, eutrophic lake with high internal phosphorus loading rates. Can. J. Fish. Aquat. Sci. 1997, 54, 2111-2120.

(41) Prepas, E. E.; Field, K. M.; Murphy, T. P.; Johnson, W. L.; Burke, J. M.; Tonn, W. M. Introduction to the Amisk Lake Project: oxygenation of a deep, eutrophic lake. Can. J. Fish. Aquat. Sci. 1997, 54, 2105-2110.

(42) Jaeger, D. Effects of hypolimnetic water aeration and iron-phosphate precipitation on the trophic level of Lake Krupunder. Hydrobiol. 1994, 275/276, 433-444.
(43) Burris, V. L.; Little, J. C. Bubble dynamics and oxygen transfer in a hypolimnetic aerator. Water Sci. Technol. 1998, 37, 293-300.

(44) Burris, V. L.; McGinnis, D. F.; Little, J. C. Predicting oxygen transfer and water flow rate in airlift aerators. Water Res. 2002, 36, 46054615.

(45) Thomas, J. A.; Funk, W. H.; Moore, B. C.; Budd, W. W. Short term changes in Newman Lake following hypolimnetic aeration with the Speece Cone. Lake Reservoir Manage. 1994, 9, 111-113.

(46) Moore, B. C.; Chen, P. H.; Funk, W. H.; Yonge, D. A model for predicting lake sediment oxygen demand following hypolimnetic aeration. Water Resour. Bull. 1996, 32, 723-731.

(47) Jung, R.; Sanders, J. O.; Lai, H. H. Improving water quality through lake oxygenation at Camanche Reservoir. In North American Lake Management Society Annual Symposium: Reno, NV, December 11999.

(48) Mobley, M. H.; Brock, W. G. Widespread oxygen bubbles to improve reservoir releases. Lake Reservoir Manage. 1995, 11, 231-234.

(49) Gibbons, H. L.; Bogus, B.; Williams, G. Worlds largest attempt at hypolimnetic aeration. Lake Reservoir Manage. 1994, 9, 76.

(50) Speece, R. E. Oxygen supplementation by Utube to the Tombigbee River. Water Sci. Technol. 1996, 34, 83-90.

(51) Gemza, A. F. Water quality improvements during hypolimnetic oxygenation in two Ontario lakes. Water Qual. Res. J. Can. 1997, 32, 365-390.

(52) Little, J. C.; McGinnis, D. F. Hypolimnetic oxygenation: Predicting performance using a discrete-bubble model. Water Sci. Technol. Water Supply 2001, 1, 185-191.

(53) Little, J. C., Personal communication.

(54) Jung, R.; Lai, H. H.; Wilczak, A. Hypolimnetic oxygenation operating experience in a eutrophic reservoir and its effect on water treatment. In Water Quality Technology Conference and Exposition: Philadelphia, PA, 2003.

(55) McGinnis, D. F.; Little, J. C. Bubble dynamics and oxygen transfer in a Speece Cone. Water Sci. Technol. 1998, 37, 285-292.

(56) Little, J. C.; Del Vecchio, D. C. Predicting water flow rate in hypolimnetic aerators. In International Association on Water Quality, Biennial Conference: Singapore, 1996.

(57) Singleton, V. L.; Gantzer, P.; Little, J. C. Linear bubble plume model for hypolimnetic oxygenation: Full-scale validation and 
sensitivity analysis. WAter Resour. Res. 2005, in press.

(58) McGinnis, D. F.; Little, J. C.; Wuest, A. Hypolimnetic oxygenation: Coupling bubbleplume and reservoir models. In Asian Waterqual 2001, First IWA Asia-Pacific Regional Conference: Fukuoka, Japan, 2001. 